\title{
On the Kinematics of Tidal Debris from Omega Cen's Progenitor Galaxy
}

\author{
Masashi Chiba ${ }^{1,3}$ and Arihiro Mizutani ${ }^{2}$ \\ ${ }^{1}$ Astronomical Institute, Tohoku University, Sendai 980-8578, Japan \\ ${ }^{2}$ National Astronomical Observatory, Mitaka, Tokyo 181-8588, Japan \\ ${ }^{3}$ E-mail: chiba@ astr.tohoku.ac.jp
}

Received 2003 October 1, accepted 2004 January 5

\begin{abstract}
The kinematic properties of tidal debris from an orbiting Galactic satellite is presented, on the assumption that its central part once contained the most massive Galactic globular cluster, $\omega$ Cen. We simulate dynamical evolution of a satellite galaxy that follows the present-day and likely past orbits of $\omega$ Cen, and analyse the kinematic nature of their tidal debris and randomly generated Galactic stars comprising spheroidal halo and flat disk components. It is found that the debris stars show a retrograde rotation at $\sim-100 \mathrm{~km} \mathrm{~s}^{-1}$, which may accord with a recently discovered stellar stream at a radial velocity of $\sim 300 \mathrm{~km} \mathrm{~s}^{-1}$ towards the Galactic longitude of $\sim 270^{\circ}$. These stars also contribute, only in part, to a reported retrograde motion of the outer halo in the north Galactic pole direction, without significantly modifying local halo kinematics near the Sun. The prospects for future debris searches and the implications for the early evolution of the Galaxy are briefly presented.
\end{abstract}

Keywords: Galaxy: formation - globular clusters: individual $(\omega$ Cen $)-$ stars: kinematics - stars: Population II

\section{Introduction}

It is well known that the most massive globular cluster in the Milky Way, $\omega$ Cen, shows unique properties in its metallicity content, internal kinematics, and structure. For instance, $\omega$ Cen shows a wide spread in metallicity unlike other Galactic globular clusters (e.g. Norris et al. 1996): its metallicity distribution is peaked at $[\mathrm{Fe} / \mathrm{H}] \simeq-1.6$, along with a second smaller peak at $[\mathrm{Fe} / \mathrm{H}] \simeq-1.2$ and a long tail extending up to $[\mathrm{Fe} / \mathrm{H}] \simeq-0.5$. Also, the metal-rich stars in $\omega$ Cen are largely enhanced in s-process elements relative to those in globular clusters and field stars with similar metallicities (e.g. Norris \& Da Costa 1995). This suggests that the ejecta from low-mass, asymptotic giant branch (AGB) stars had to be retained and incorporated into the next-generation stars. However, although $\omega$ Cen is massive $\left(5 \times 10^{6} \mathrm{M}_{\odot}\right)$, it is unable to retain the AGB ejecta, as shown by Gnedin et al. (2002). Thus, an isolated formation of $\omega$ Cen is unlikely, because the enriched gas would easily be lost by encountering the Galactic disk. The most viable explanation for the uniqueness of $\omega$ Cen is that it was once the dense nucleus of a dwarf galaxy, i.e. a nucleated dwarf (Freeman 1993). A gravitational potential of the progenitor's dark matter would help retain the enriched gas and let the cluster be self-enriched at least over a few Gyr.

In this contribution, we pursue the possible kinematical evidence for the existence of such a dwarf galaxy which had disrupted in the past. Dinescu (2002) first investigated this issue, by examining the possible signature of the progenitor's tidal debris among nearby metal-poor stars in the catalogue of Beers et al. (2000, hereafter B00). They identified a group of stars with $-2.0<[\mathrm{Fe} / \mathrm{H}] \leq-1.5$, which departs from the characteristics of the inner Galactic halo, but has retrograde orbits similar to $\omega$ Cen. Their simplified disruption model of the progenitor galaxy demonstrated that trailing tidal debris, with orbital characteristics similar to the cluster, can be found in the solar neighbourhood, although the concrete spatial distribution and kinematics of the debris stars remain yet unclear.

Dinescu's (2002) work motivates us to undertake a more refined approach to the issue, i.e. to conduct an $N$-body simulation for the tidal disruption of $\omega$ Cen's progenitor galaxy (Mizutani et al. 2003). We obtain the characteristic structure and kinematics of its debris stars and compare them with various observations showing signatures of recent merging events in the Milky Way (Gilmore et al. 2002, hereafter GWN; Kinman et al. 2003, hereafter K03; Chiba \& Beers 2000, hereafter CB). In particular, we show that a recently identified stream of stars at a radial velocity of $\sim 300 \mathrm{~km} \mathrm{~s}^{-1}$ (GWN) is a natural outcome of the current disruption model, without significantly modifying local halo kinematics near the Sun.

\section{Model of a Progenitor Galaxy}

For the purpose of enlightening the characteristics of tidal debris, we first assume a fixed external gravitational potential (representing the Milky Way) for the dynamical evolution of an orbiting dwarf galaxy. The potential consists of a spherical Hernquist bulge $\Phi_{\mathrm{b}}(r)$, a MiyamotoNagai disk $\Phi_{\mathrm{d}}(R, z)$, and a logarithmic dark halo $\Phi_{\mathrm{h}}(r)$, where $r$ is the Galactocentric distance and $(R, z)$ denotes 
cylindrical coordinates. Each is given as $\Phi_{\mathrm{b}}(r)=-G M_{\mathrm{b}} /$ $(r+a)$,

$$
\Phi_{\mathrm{d}}(R, z)=-G M_{\mathrm{d}} / \sqrt{R^{2}+\left(b+\sqrt{z^{2}+c^{2}}\right)^{2}},
$$

and $\Phi_{\mathrm{h}}(r)=v_{\mathrm{h}}^{2} / 2 \ln \left(r^{2}+d^{2}\right)$, where $M_{\mathrm{b}}=3.4 \times 10^{10} \mathrm{M}_{\odot}$, $a=0.7 \mathrm{kpc}, M_{\mathrm{d}}=10^{11} \mathrm{M}_{\odot}, b=6.5 \mathrm{kpc}, c=0.26 \mathrm{kpc}$, $v_{\mathrm{h}}=186 \mathrm{~km} \mathrm{~s}^{-1}$, and $d=12 \mathrm{kpc}$. This choice yields a circular velocity of $228 \mathrm{~km} \mathrm{~s}^{-1}$ at the solar circle of $R_{\odot}=8 \mathrm{kpc}$ and a flat rotation curve outside $R_{\odot}$. On our adoption of a logarithmic dark halo, we note that while a dark halo may be modelled by the universal profile proposed by Navarro et al. (1997, NFW profile), such a profile as obtained by $N$-body simulations can be modified by the inclusion of baryonic gas, mostly in the inner part of a galaxy where its density is increased owing to baryonic infall, in such a manner that the density profile may be changed to an isothermal one (e.g. Barnes 1987). It is also noted that the adoption of the NFW profile does not affect our results, as the inner density profile of concern is dominated by the bulge and disk components. A dwarf galaxy orbiting in this galactic potential is then represented by self-gravitating particles following a King (1966) model, where the central density, central velocity dispersion, and core radius are given as $0.3 \mathrm{M}_{\odot} \mathrm{pc}^{-3}$, $18.1 \mathrm{~km} \mathrm{~s}^{-1}$, and $0.56 \mathrm{kpc}$, respectively. In addition, a particle with a mass of $5 \times 10^{6} \mathrm{M}_{\odot}$ representing $\omega$ Cen is placed at the centre of the galaxy. This setting yields the total mass of the system as $M_{\mathrm{tot}}=5.79 \times 10^{8} \mathrm{M}_{\odot}$. On the other hand, the total mass of stars in this hypothetical galaxy is estimated from the mean metallicity of stars in $\omega \mathrm{Cen}(\langle[\mathrm{Fe} / \mathrm{H}]\rangle \sim-1.6)$, combined with the metallicityluminosity relation for the Local Group dwarfs (Côté et al. 2000) and the mass-to-light ratio (assuming $M / L \sim 4$ ), yielding $M_{\text {stars }} \sim 10^{7} \mathrm{M}_{\odot}$. Thus, the mass of the simulated galaxy is largely dominated by dark matter. The model galaxy is represented by a collection of $10^{4}$ particles and the self-gravity is calculated in terms of a multiple expansion of the internal potential to fourth order (White 1983; Zaritsky \& White 1988).

This model dwarf is disrupted by Galactic tides in the course of its orbital motion, whereas its dense core is expected to survive and follow $\omega$ Cen's orbit. We examine two representative orbits for the progenitor, models 1 and 2: model 1 follows the current orbit of $\omega$ Cen, whereas for model 2 , we calculate an orbit back to the past over $\sim 2 \mathrm{Gyr}$ from its current position and velocity taking into account dynamical friction, and then set a progenitor galaxy on its non-decaying orbit. These two models provide us with satisfactory information on the generic properties of a tidally disrupted progenitor, and we postulate that the realistic nature of their debris is midway between these model predictions. We calculate $\omega$ Cen's orbit, based on the current distance $D=5.3 \pm 0.5 \mathrm{kpc}$ from the Sun, position $(l, b)=\left(309^{\circ}, 15^{\circ}\right)$, proper motion $\left(\mu_{\alpha} \cos \delta, \mu_{\delta}\right)=(-5.08 \pm 0.35,-3.57 \pm 0.34) \mathrm{mas} \mathrm{yr}^{-1}$, and heliocentric radial velocity $v_{\text {los }}=232.5 \pm 0.7 \mathrm{~km} \mathrm{~s}^{-1}$ (Dinescu et al. 1999, hereafter DGvA). This orbit for

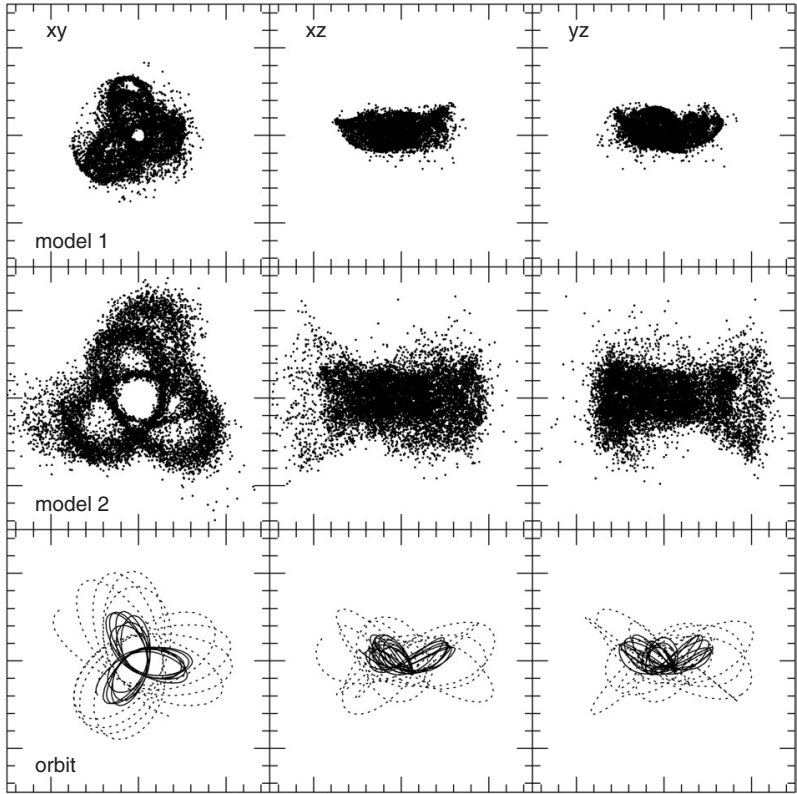

Figure 1 Upper (middle) panel shows the spatial distribution of the tidally disrupted debris for model 1 (model 2) after the 1.37 (1.86) Gyr orbital excursion of $\omega$ Cen's progenitor galaxy. Lower panel shows the orbit of the galaxy centre for model 1 (solid line) and model 2 (dotted line). The plots are projected onto three orthogonal planes, where the Sun is located at $x=-8 \mathrm{kpc}$ and $x y$ corresponds to the disk plane. A frame measures $15 \mathrm{kpc}$ on a side of each panel. The current position of $\omega$ Cen is at $(x, y, z)=(-4.8,-4.0,1.4) \mathrm{kpc}$.

model 1 is characterised by frequent disk crossings with a period of $\tau_{\mathrm{orb}}=0.8 \times 10^{8} \mathrm{yr}$, retrograde motion, and apocentric and pericentric distances $\left(r_{\text {apo }}, r_{\text {peri }}\right)=$ $(6.4,1.1) \mathrm{kpc}$. For model 2 , we obtain $\tau_{\mathrm{orb}}=1.5 \times 10^{8} \mathrm{yr}$ and $\left(r_{\text {apo }}, r_{\text {peri }}\right)=(11.3,3.0) \mathrm{kpc}$. In both experiments, we place a progenitor galaxy at apocentre to maximise its survival chances.

We plot the spatial distribution of the tidally disrupted debris in Figure 1. The upper (middle) panel shows model 1 (model 2) after the 1.37 (1.86) Gyr orbital excursion of the progenitor galaxy. The lower panel shows the orbit of the galaxy centre. A rosette-like feature of the debris becomes steady after about eight orbital periods. Model 1 results in more compact distribution than model 2, which reflects the difference in orbital radii. Figure 2 shows the velocity distributions of the debris particles in cylindrical coordinates $\left(v_{R}, v_{\phi}, v_{z}\right)$. As is evident, both models provide essentially the same debris kinematics: most notorious is a sharply peaked $v_{\phi}$ distribution at $\sim-100 \mathrm{~km} \mathrm{~s}^{-1}$, arising from a retrograde orbit of a progenitor. These kinematics suggest that the difference between models 1 and 2 resides only in the spatial extent of the debris.

\section{Kinematics of Stream and Galactic Stars}

\subsection{Method}

We analyse the kinematics of both the simulated debris and other Galactic stars generated randomly by a Monte Carlo method. The metal-poor halo is modelled as a flattened spheroid $\rho \propto\left(R^{2}+z^{2} / q^{2}\right)^{-3.5 / 2}$, where $q$ is an 


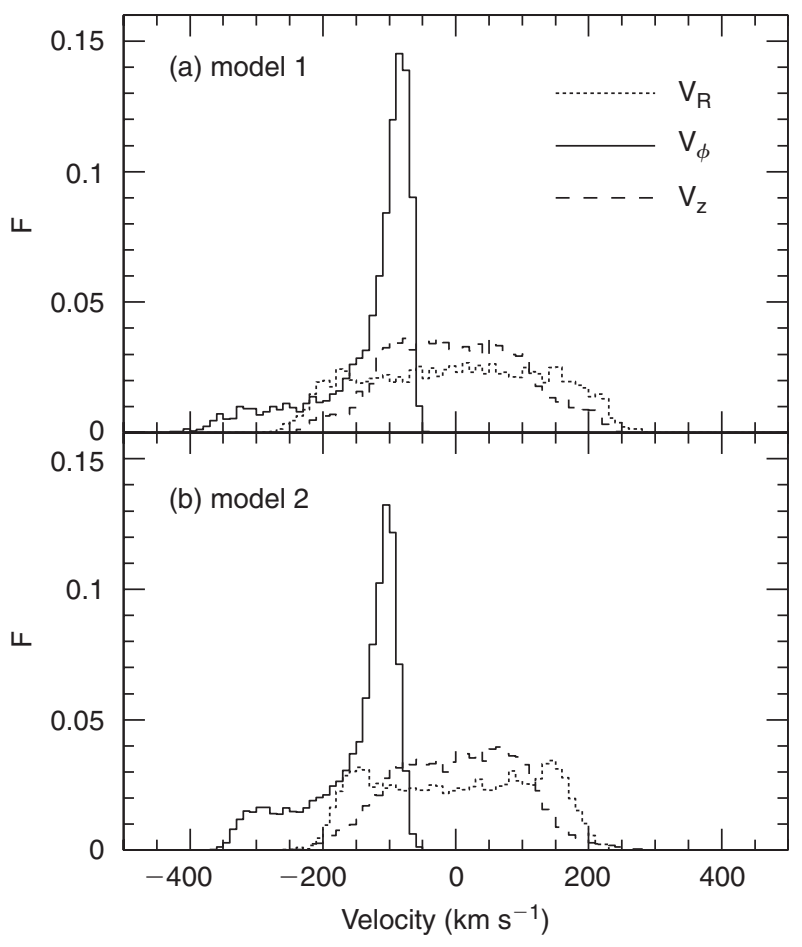

Figure 2 Velocity distribution of the debris particles in cylindrical coordinates, $v_{R}$ (dotted), $v_{\phi}$ (solid), $v_{z}$ (dashed), for model 1 (a) and 2 (b).

axis ratio in the range $0.55-0.7$, anisotropic velocity ellipsoid $\left(\sigma_{R}, \sigma_{\phi}, \sigma_{z}\right)=(154,121,96) \mathrm{km} \mathrm{s}^{-1}$, and small mean rotation $\left\langle v_{\phi}\right\rangle=24 \mathrm{~km} \mathrm{~s}^{-1}$, as found for halo stars with $[\mathrm{Fe} / \mathrm{H}]<-2$ near the Sun $(\mathrm{CB})$. Thin and thick disks are modelled as $\rho \propto \exp \left(-R / R_{\mathrm{d}}\right) \sec ^{2}\left(z / z_{\mathrm{d}}\right)$, where $R_{\mathrm{d}}=3.5 \mathrm{kpc}$ and $z_{\mathrm{d}}=0.3$ (1) kpc for a thin (thick) disk. Both disks rotate at $200 \mathrm{~km} \mathrm{~s}^{-1}$, having velocity ellipsoids of $(34,25,20) \mathrm{km} \mathrm{s}^{-1}$ and $(46,50,35) \mathrm{km} \mathrm{s}^{-1}$ for thin and thick disks, respectively $(\mathrm{CB})$. The relative fraction of each component is fixed using observed local densities near the Sun, in such a manner that the halo and thick-disk densities at $D<1 \mathrm{kpc}$ are $0.2 \%$ and $2 \%$ of the thin-disk density, respectively (Yamagata \& Yoshii 1992).

In our model of $\omega$ Cen's progenitor galaxy, the selfgravitating particles represent both stars and dark matter particles. We note that the correct conversion of the mass of the simulated system into that of the presumed stellar system alone remains yet uncertain, because the correct $M / L$ ratio of stars as well as the amount of dark matter in the progenitor galaxy is unavailable. As a useful model parameter to incorporate this ambiguity for the current kinematic analysis, we set a quantity $f$ as the fraction of the debris particles relative to halo stars near the Sun, so that the normalisation of the halo density is fixed for the given number of the neighbouring debris particles and $f$.

A typical value of $f$ for the conversion of the simulated particles to stars is estimated in the following manner. Model 1 (model 2) yields 21 (74) particles at $D<2 \mathrm{kpc}$, giving the mass density $\rho_{g}=0.4(1.3) \times 10^{-4} \mathrm{M}_{\odot} \mathrm{pc}^{-3}$ near the Sun, whereas the total mass density and metalpoor halo density have been derived as $8 \times 10^{-3} \mathrm{M}_{\odot} \mathrm{pc}^{-3}$

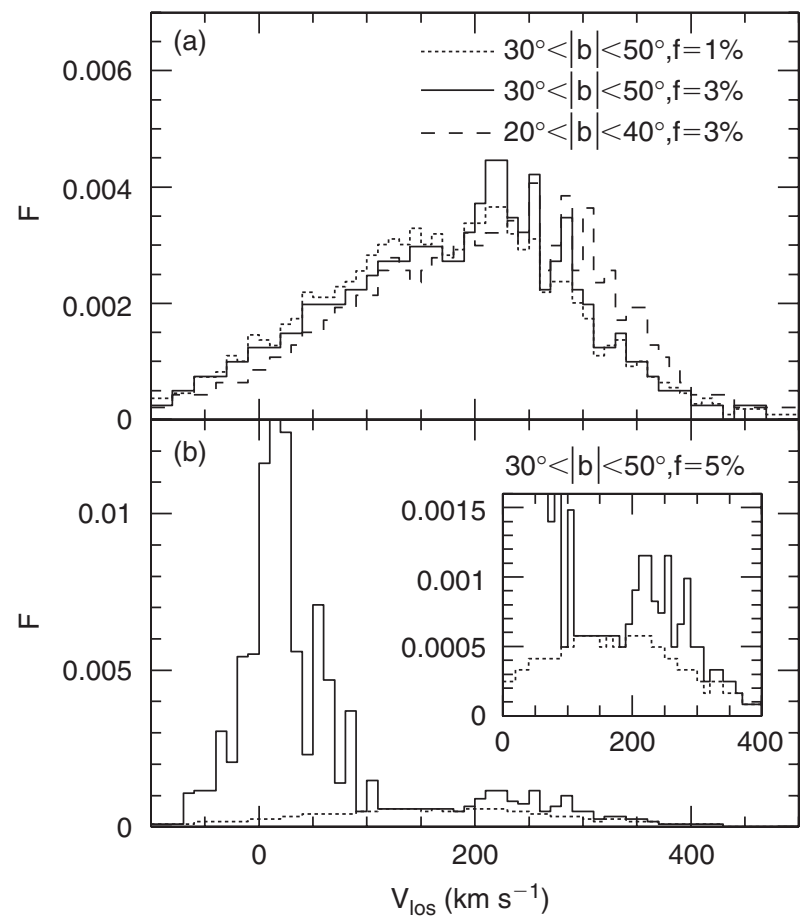

Figure 3 (a) Distribution of the heliocentric radial velocities in the direction of the GWN survey, for the debris stars of model 2 and randomly generated halo stars with $q=0.7$. We select the stars at $1<D<5 \mathrm{kpc}$ and $260^{\circ}<l<280^{\circ}$ in the fields $30^{\circ}<|b|<50^{\circ}$ (with $f=1 \%$ and $3 \%$ for dotted and solid histograms, respectively) and $20^{\circ}<|b|<40^{\circ}$ (with $f=3 \%$, dashed histogram). (b) The same as (a) but incorporating the randomly generated disk stars as well for $30^{\circ}<|b|<50^{\circ}$ with $f=5 \%$ (solid histogram). The dotted histogram denotes the contribution from the metal-poor halo alone.

(Gates et al. 1995) and $6.4 \times 10^{-5} \mathrm{M}_{\odot} \mathrm{pc}^{-3}$ (Gould et al. 1998), respectively. Then, if the debris stars (with $M_{\text {stars }} \sim 10^{7} \mathrm{M}_{\odot}$ ) are distributed in the same manner as the simulated particles (with $M_{\text {tot }}=5.74 \times 10^{8} \mathrm{M}_{\odot}$ ), which would be a reasonable approximation in view of the dissipationless nature of stars, the mass density of the debris stars in the solar neighbourhood can be estimated as $\left(M_{\text {stars }} / M_{\text {tot }}\right) \rho_{g}=O\left(10^{-6}\right) \mathrm{M}_{\odot} \mathrm{pc}^{-3}$, which is about $1 \%$ of the halo density. Thus, $f$, defined here at $D<2 \mathrm{kpc}$, is expected to be of the order of a few per cent.

\subsection{Radial Velocity Distribution at $1 \simeq 270^{\circ}$}

GWN recently reported their spectroscopic survey of $\sim 2000 \mathrm{~F} / \mathrm{G}$ stars down to $V=19.5$ magnitudes, in the direction against Galactic rotation $(l, b)=\left(270^{\circ},-45^{\circ}\right)$ and $\left(270^{\circ},+33^{\circ}\right)$, where radial velocities in combination with distances largely reflect orbital angular momentum. Their calibration of the stars at distances of a few kpc from the Sun leads to the discovery of two stellar streams at $v_{\text {los }} \sim 100 \mathrm{~km} \mathrm{~s}^{-1}$ and $\sim 300 \mathrm{~km} \mathrm{~s}^{-1}$, which are not explained by any existing Galactic components (see their Figures 2 and 3). While the stream at $v_{\text {los }} \sim 100 \mathrm{~km}^{-1}$ was reproduced by their model of a merging satellite in prograde rotation, the stream at $v_{\text {los }} \sim 300 \mathrm{~km}^{-1}$ remains yet unexplained. 
Figure 3(a) shows the $v_{\text {los }}$ distribution for the simulated debris of model 2 and halo stars of $q=0.7$ (i.e. without disks) at $1<D<5 \mathrm{kpc}, 260^{\circ}<l<280^{\circ}$, and two fields for $b$. Figure $3(\mathrm{~b})$ shows the distribution when disk stars are incorporated. As is evident, the debris stars from $\omega$ Cen's progenitor galaxy form a local peak at $v_{\text {los }} \sim 300 \mathrm{~km} \mathrm{~s}^{-1}$, which is provided by many stars having $v_{\phi} \sim-100 \mathrm{~km} \mathrm{~s}^{-1}$ (Figure 2). This is in good agreement with the $v_{\text {los }} \sim 300 \mathrm{~km} \mathrm{~s}^{-1}$ stream discovered by GWN. A more flattened halo than the case $q=0.7$, which is possible within a range of $0.55 \leq q \leq 0.7$ (CB), yields a higher peak, since the density contrast of the debris relative to the halo is made higher in this survey region. We also make a plot for model 1 but do not present it here as it is essentially the same as Figure 3 for model 2. This reflects that model 1 shows the same velocity distribution as model 2 (Figure 2), although to attain the same peak height at $\sim 300 \mathrm{~km} \mathrm{~s}^{-1}$, $f$ must be a few factors larger and the selected range of $l$ a few degrees higher than the respective values in model 2 , because of a smaller number of debris particles near the Sun. This rule applies to other considerations, below, as well.

\subsection{Kinematics at the North Galactic Pole}

The halo kinematics at the north Galactic pole (NGP) deserve special attention. Majewski (1992) suggested that the outer halo at the NGP shows a retrograde rotation $\left\langle v_{\phi}\right\rangle \sim-55 \mathrm{~km} \mathrm{~s}^{-1}$ at $z>4 \mathrm{kpc}$. Also, K03 reported from their analysis of RR Lyrae and blue horizontal branch stars that the halo at $2<z<12 \mathrm{kpc}$ shows a retrograde rotation at $\left\langle v_{\phi}\right\rangle \sim-65 \mathrm{~km} \mathrm{~s}^{-1}$. On the other hand, halo stars near the Sun show no significant retrograde rotation (CB).

For the purpose of elucidating the effect of the debris stars on the NGP kinematics, we select the debris of model 2 and randomly generated stars at $b>70^{\circ}$ and $2<$ $D<5 \mathrm{kpc}$ (resembling K03's selection). Since the observational determination of full space velocities involves rather inaccurate information of proper motions compared to radial velocities, we convolve the velocity distribution of stars with a Gaussian distribution for velocity errors, having $1 \sigma$ of a typical $30 \mathrm{~km} \mathrm{~s}^{-1}$ error. Figure 4 shows the velocity distributions at various values of $f$. It follows that the debris stars provide a non-Gaussian feature in the velocity distribution. The $v_{\phi}$ distribution shows an extra peak at $\sim-100 \mathrm{~km} \mathrm{~s}^{-1}$, whereby the debris stars decrease $\left\langle v_{\phi}\right\rangle$ from that of a pure halo sample. However, the change of $\left\langle v_{\phi}\right\rangle$ by the inclusion of the debris stars with $f=5 \%$ amounts to only $-19(-14) \mathrm{km} \mathrm{s}^{-1}$ for $q=0.55(0.7)$, which are still insufficient to explain the reported $\left\langle v_{\phi}\right\rangle=-35 \sim-75 \mathrm{~km} \mathrm{~s}^{-1}$. For the $v_{R}$ and $v_{z}$ distributions, their means for the case of $q=0.7$ and $f=5 \%$ are slightly increased to $\left\langle v_{R}\right\rangle=7 \mathrm{~km} \mathrm{~s}^{-1}$ and $\left\langle v_{z}\right\rangle=3 \mathrm{~km} \mathrm{~s}^{-1}$, respectively, whereas the velocity dispersions are decreased by $6 \mathrm{~km} \mathrm{~s}^{-1}$ and $5 \mathrm{~km} \mathrm{~s}^{-1}$, respectively, when the debris stars are considered. Note that if we extend the selection of the stars to higher $z$ or instead consider model 1 , the changes in $\left\langle v_{\phi}\right\rangle$ become smaller than the above-mentioned values, because there are no debris

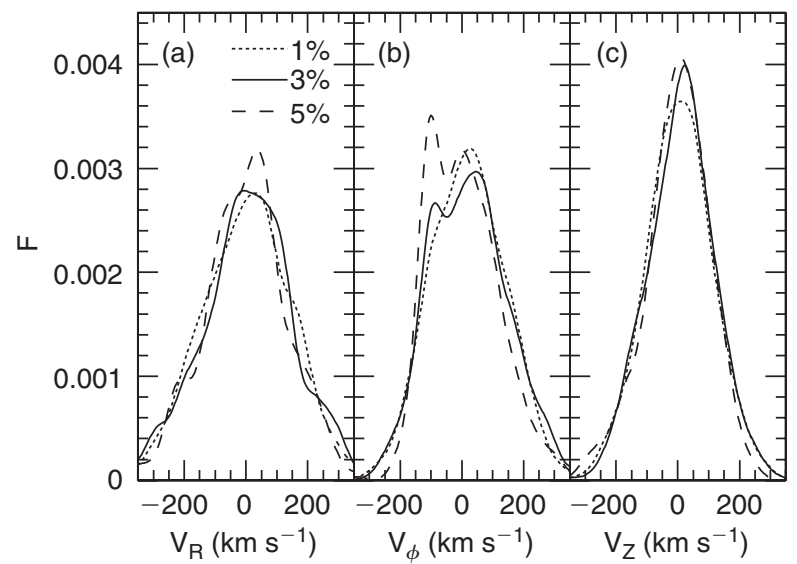

Figure 4 Velocity distribution of the simulated stars of model 2 in the NGP direction. In this plot, we select the stars at $b>70^{\circ}$ and $2<D<5 \mathrm{kpc}$ and convolve their derived velocity distribution with a Gaussian error distribution having $1 \sigma$ of $30 \mathrm{~km} \mathrm{~s}^{-1}$. The axis ratio of the metal-poor halo is set as $q=0.7$. Dotted, solid, and dashed lines denote $f=1 \%, 3 \%$, and $5 \%$, respectively.

stars in our current model (Figure 1). Thus, it is safe to conclude that the debris stars contribute only in part to a reported retrograde motion at the NGP.

\subsection{Local Halo Kinematics}

We select the simulated stars at the cylindrical coordinates of $6.5<R<9.5 \mathrm{kpc}$ and $z<4 \mathrm{kpc}$ and at a distance from the Sun of $D<4 \mathrm{kpc}$ (as was drawn by CB), convolve the velocities with a Gaussian error distribution of $1 \sigma=30 \mathrm{~km} \mathrm{~s}^{-1}$, and compare with corresponding stars with $[\mathrm{Fe} / \mathrm{H}] \leq-2$ in B00. It follows that the non-Gaussian feature of the velocity distribution is much weaker than that at the NGP: the change of $\left\langle v_{\phi}\right\rangle$ for $f=5 \%$ is only $-9(-10) \mathrm{km} \mathrm{s}^{-1}$ in model 1 (model 2). This is due to the characteristic debris distribution, where the number density near $z=0$ is smaller than at high $|z|$ (Figure 1).

\section{Discussion}

Our simple model of an orbiting dwarf galaxy that once contained $\omega$ Cen predicts a sequence of tidal streams in retrograde rotation and their existence can be imprinted in the kinematics of nearby stars, especially in the direction against Galactic rotation (GWN) and at the NGP (K03), while local halo kinematics remain unchanged. The simulated streams are mostly distributed inside the solar circle, as suggested from the current orbital motion of $\omega$ Cen (DGvA; Dinescu 2002). In contrast to the Sagittarius dwarf galaxy having a polar orbit (Ibata et al. 1997), the orbit of $\omega$ Cen's progenitor galaxy is largely affected by a non-spherical disk potential, where the orbital plane exhibits precession with respect to the Galactic pole, causing self-crossing of tidal streams in the disk region (Figure 1). The projection of the orbit perpendicular to the disk plane shows an ' $\mathrm{X}$ '-like feature, thereby leaving denser streams at high $|z|$ than at low $|z|$ for a given radius. These characteristic spatial distributions of the debris 
stars give rise to more significant effects of the debris at the NGP than in the solar neighbourhood, as shown here, although the current simulation failed to reproduce the reported largely retrograde rotation at the NGP from the $\omega$ Cen debris alone; perhaps other, yet unknown, halo substructures must be considered to reproduce the observations.

Existing kinematic studies of Galactic stars to search for a signature of $\omega$ Cen's progenitor galaxy are yet confined to nearby stars, where the significance of the debris streams is modest, as shown here. Searches of stars inside the solar circle are more encouraging (Figure 1), in particular in the directions of $l \sim 320^{\circ}$ and $l \sim 50^{\circ}$, where we expect the presence of high-velocity streams at $v_{\text {los }}=200 \sim 300 \mathrm{~km} \mathrm{~s}^{-1}$ and $-400 \sim-300 \mathrm{~km} \mathrm{~s}^{-1}$, respectively. Future radial velocity surveys of these fields, including the sample of the Sloan Digital Sky Survey or the planned Radial Velocity Experiment, are worth exploring in this respect. Also, detailed abundance studies of candidate stream stars will be intriguing, because such stars may exhibit different abundance patterns from field halo stars, as found in dwarf galaxies (Shetrone et al. 2001).

In this work, we adopt a fixed external gravitational potential for the calculation of an orbiting dwarf galaxy and its dynamical evolution, thereby neglecting dynamical friction against the satellite. However, to set more refined limits on its dynamical history, it is required to fully take into account frictional effects on the orbit as well as dynamical feedback of a satellite on the structure of the Galactic disk. For instance, Tsuchiya et al. (2003) reported their numerical models for a satellite with strong orbital decay and succeeded in reproducing the current orbit of $\omega$ Cen from the launch of the progenitor satellite at $58 \mathrm{kpc}$ from the Galactic centre. They also showed that the debris particles at $3 \mathrm{Gyr}$ are already smeared out and distributed in a flattened disk without having significant stream-like features as obtained here. It is noted that whether or not stream-like features survive to the current epoch depends on when and how a satellite galaxy is disrupted by Galactic tides, or, in other words, the observational information on such features and the comparison with simulation results are useful for placing important constraints on when a satellite merging occurs. For this purpose, we are currently undertaking full $\mathrm{N}$-body simulations of several host-satellite systems using GRAPE5, where both host and satellite galaxies are represented by lively dark halos and stellar components. Our goals with the use of GRAPE5 are to set tight limits on the initial total mass, internal mass distribution, and orbital motion of a progenitor galaxy, as well as the timing of merging with the Galactic disk, based on the comparison between the observation (e.g. RAVE) and simulation results for stream-like structures in the Milky Way. More details will be reported elsewhere (A. Mizutani \& M. Chiba, in preparation).

\section{Acknowledgements}

M.C. thanks Kenji Bekki and Tim Beers for useful discussions. We are grateful to anonymous referees for invaluable comments on the manuscript.

\section{References}

Barnes, J. E. 1987, in Nearly Normal Galaxies: From the Planck Time to the Present, ed. S. Faber (New York: Springer-Verlag), 154

Beers, T. C., Chiba, M., Yoshii, Y., Platais, I., Hanson, R. B., Fuchs, B., \& Rossi, S. 2000, AJ, 119, 2866 (B00)

Chiba, M., \& Beers, T. C. 2000, AJ, 119, 2843 (CB)

Côté, P., Marzke, R. O., West, M. J., \& Minniti, D. 2000, ApJ, 533,869

Dinescu, D. I. 2002, in Omega Centauri: A Unique Window into Astrophysics, ASP Conf. Series 265, eds. F. van Leeuwen, J. D. Hughes, \& G. Piotto (ASP: San Francisco), 365

Dinescu, D. I., Girard T. M., \& van Altena, W. F. 1999, AJ, 117, 1792 (DGvA)

Freeman, K. C. 1993, in The Globular Cluster-Galaxy Connection, ASP Conf. Series 48, eds. G. H. Smith, \& J. P. Brodie (ASP: San Francisco), 608

Gates, E. I., Gyuk, G., \& Turner, M. S. 1995, ApJ, 449, L123

Gilmore, G., Wyse, R. F. G., \& Norris, J. E. 2002, ApJ, 574, L39 (GWN)

Gnedin, O. Y., Zhao, H.-S., Pringle, J. E., Fall, S. M., Livio, M., \& Meylan, G. 2002, ApJ, 568, L23

Gould, A., Flynn, C., \& Bahcall, J. N. 1998, ApJ, 503, 798

Ibata, R. A., Wyse, R. F. G., Gilmore, G., Irwin, M. J., \& Suntzeff, N. B. 1997, AJ, 113, 634

King, I. R. 1966, AJ, 71, 64

Kinman, T. D., Cacciari, C., Bragaglia, A., Buzzoni, A., \& Spagna, A. 2003, in Galactic Dynamics Workshop, EAS, 10, 115

Majewski, S. R. 1992, ApJS, 78, 87

Mizutani, A., Chiba, M., \& Sakamoto, T. 2003, ApJ, 589, L89

Navarro, J. F., Frenk, C. S., \& White, S. D. M. 1997, ApJ, 490, 493

Norris, J. E., \& Da Costa, G. S. 1995, ApJ, 447, 680

Norris, J. E., Freeman, K. C., \& Mighell, K. L. 1996, ApJ, 462, 241

Shetrone, M. D., Côté, P., \& Sargent, W. L. W. 2001, ApJ, 548, 592

Tsuchiya, T., Dinescu, D. I., \& Korchagin, V. I. 2003, ApJ, 589, L29

White, S. D. M. 1983, ApJ, 274, 53

Yamagata, T., \& Yoshii, Y. 1992, AJ, 103, 117

Zaritsky, D., \& White, S. D. M. 1988, MNRAS, 235, 289 